\title{
Identification of Galliformes through Forensically Informative Nucleotide Sequencing (FINS) and its Implication in Wildlife Forensics
}

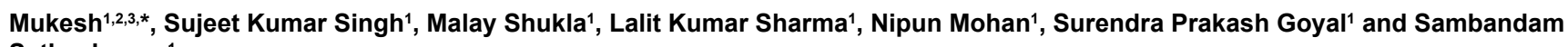
Sathyakumar ${ }^{1}$

${ }^{1}$ Wildlife Institute of India, P. O. Box 18, Chandrabani, Dehradun 248 001, Uttarakhand, India

${ }^{2}$ Department of Biotechnology, Kurukshetra University, Kurukshetra- 136 119, Haryana, India

${ }^{3}$ Animal Biotechnology Centre, National Dairy Research Institute, Karnal- 132 001, Haryana, India

\begin{abstract}
Galliformes are hunted for the demand of their attractive feathers and to supply a cheap animal food for the rural communities. In such cases, species identification through visual inspection of the meat or based on feather morphometrics is a challenging task for the law enforcement agencies to enforce the Wildlife Protection Act (WPA) Here, we extracted DNA from the individual feathers of unknown species encountered during field surveys and two mitochondrial genes (12S rRNA and Cytochrome $b$ ) were amplified using universal primers for species identification. Most homologous sequences were retrieved using NCBI-BLAST for each generated sequence of both the genes. Neighbor-Joining trees based on Kimura 2 parameter distance matrices in FINS analysis identified the species from the individual feather with strong bootstrap support. Nine species specific polymorphic sites were found in the partial sequence of Cytochrome $b$ gene that differentiated Pavo cristatus to Pavo muticus imperator. Our study highlighted the importance of feathers in identifying the species and their applicability in wildlife offence cases using FINS approach.
\end{abstract}

Keywords: Galliformes; FINS; $12 S$ rRNA; Cytochrome b; Species identification

\section{Introduction}

Galliformes that commonly referred as a 'gallinaceous birds' are popular for their attractive bright plumage, shy and elusive behavior. They comprise of 70 genera and 284 species worldwide [1]. In India, 45 species of galliformes have been reported which includes one megapode, 27 partridges, quails, francolins and snow cocks and 17 pheasants. Of these, seven species are endemic to India and the global status of 12 species is categorized as 'threatened'. This is largely due to habitat loss, degradation and poaching [2]. In India, illegal poaching of birds is silently on in many protected areas because of the demand of their colorful feathers and cheap source of animal protein for the rural communities that live nearby the Protected Areas. Hunters kill birds for meat and trade their feathers resulting to leave the young orphan chicks to sustain alone in the forest that cause a significant decline in galliformes population across their distribution range in India. Lack of stringent measures to put a check on their poaching is making the situation worst. Therefore, the problem becomes amplified for the law enforcement agencies who are involved in determining the species of the seized material to enforce the wildlife protection act. In past, species identification is generally carried out by immunological methods [3] but with the advancement of the new technologies, nowadays, both nuclear and mitochondrial genes have been targeted for species identification. Highly conserved species specific mitochondrial genes viz. $12 S$ rRNA, Cytochrome $b$ and 16S rRNA are amplified using universal primers for identifying species from the seized biological material [4-10]. This approach is popularized as 'Forensically Informative Nucleotide Sequencing (FINS)' $[11,12]$. In the present study, we tested individual feathers for amplification of $12 S$ rRNA and Cytochrome $b$ genes and their applicability in species identification using FINS approach.

\section{Materials and Methods}

\section{DNA extraction and PCR amplification}

Fallen feathers from 24 birds of unknown species and pulled feathers from a dead Blood Pheasant (Ithaginis cruentus), were collected during field surveys (2008-2010) in different Protected Areas of Uttarakhand state. Feathers of a Silver Pheasant (Lophura nycthemera) were collected from a captive bird that was kept for display in Bharat Ratna Pt. Govind Ballabh Pant High Altitude Zoo, Nainital, Uttarakhand. DNA was extracted from the individual feather follicle $(c a .0 .5-1 \mathrm{~cm})$ which remained attached to the calamus of the individual feather using Qiagen DNeasy tissue kit (Qiagen, Germany) following manufacturer's protocol with slight modifications as suggested by us elsewhere [13]. Two mitochondrial markers viz. $12 S$ rRNA and Cytochrome b genes were amplified using the universal primers [14]. All PCR reactions were performed on Applied Biosystems thermal cycler (ABI, 2720) in a reaction volume of $10 \mu \mathrm{l}$ containing $1 \mathrm{X}$ PCR buffer $(50 \mathrm{mM} \mathrm{KCl}$, $10 \mathrm{mM}$ tris- $\mathrm{HCl}$ ), $2.5 \mathrm{mM}$ of $\mathrm{MgCl}_{2}, 200 \mu \mathrm{M}$ of each d-NTP, $1.25 \mu \mathrm{g}$ BSA, 4 pM of each primer and $0.5 \mathrm{U}$ of Taq DNA polymerase (MBI, Fermentas) and approximately 15-20 ng of genomic DNA. The PCR cycling conditions were as follows: initial denaturation at $94^{\circ} \mathrm{C}$ for 2 mins, followed by 35 cycle of denaturation at $94^{\circ} \mathrm{C}$ for $1 \mathrm{~min}$, primer annealing at $50^{\circ} \mathrm{C}$ for $1 \mathrm{~min}$, primer extension at $72^{\circ} \mathrm{C}$ for $1.5 \mathrm{~min}$. with a final extension at $72^{\circ} \mathrm{C}$ for $10 \mathrm{~min}$. After amplification, $4 \mu \mathrm{l}$ of PCR products were subjected to electrophoresis on $2 \%$ agarose gel and visualized over transilluminator to detect the amplification.

*Corresponding author: Dr. Mukesh, Animal Biotechnology Centre, National Dairy Research Institute, Karnal-132001, Haryana, India, E-mail: thamukesh@ gmail.com

Received December 17, 2012; Accepted December 26, 2012; Published December 28, 2012

Citation: Mukesh, Singh SK, Shukla M, Sharma LK, Mohan N, et al. (2013) Identification of Galliformes through Forensically Informative Nucleotide Sequencing (FINS) and its Implication in Wildlife Forensics. J Forensic Res 4: 195 doi:10.4172/2157-7145.1000195

Copyright: $(2013$ Mukesh, et al. This is an open-access article distributed under the terms of the Creative Commons Attribution License, which permits unrestricted use, distribution, and reproduction in any medium, provided the original author and source are credited. 


\section{DNA sequencing}

The PCR products were cleaned up using Exo-SAP treatment to remove residual oligonucleotides and dNTPs prior to DNA sequencing. Forward primer of the universal primers of $12 S$ rRNA (12SFwd 5'-AAAAAGCTTCAAACTGGGATTAGATACCCCACTAT-3') and Cytochrome b gene (CytbFwd 5'-AAAAAGCTTCCATCCAACATCTCAGCATGATGAAA-3') were used for setting up the cycle sequencing reaction using the Big dye terminator cycle sequencing kit $v$ 3.1. The sequencing products were cleaned up to remove any unbound ddNTPs using alcoholic precipitation method and subjected for sequencing to ABI 3130 Genetic Analyzer (Applied Biosystems).

\section{Sequence analysis}

Qualities of sequences were determined using Sequence Analysis v 5.2 software (Applied Biosystems) and validated by Sequencher v 4.7 software (www. genecode. com). All good quality sequences were compared with NCBI/GenBank (http://www. ncbi. nlm. nih. gov/) database using BLAST tool and most homologous sequences were retrieved from NCBI database. Multiple sequence alignment (MSA) was performed using CLUSTAL W as implemented in BioEdit v 7.0.9.0 software [15]. The phylogenetic trees were generated based on Kimura 2 parameter distance matrix for both the genes using neighbor joining method for all the aligned sequences in Mega v 5.0 [16]. The complete mitochondrial genome of G. g. murghi (GU261709.1) was retrieved from NCBI database and aligned with the sequences generated in the present study to identify the species specific polymorphic sites or SNPs.

\section{Results and Discussion}

Out of 24 different feather samples, good quality DNA could be extracted from 10 samples and rest samples probably might have suffered by microbial activity prior to sampling, therefore, they did not yield enough DNA. Seven feather samples showed visible bands and both the mitochondrial genes, $12 S$ rRNA and Cytochrome $b$ were sequenced for three feather samples (Table 1).

Feathers 'B', 'C', 'D' and 'F' were identified using $12 S$ rRNA gene as Gallus gallus murghi, Francolinus pondicerianus, Pavo cristatus and Lophura nycthemera, respectively with strong bootstrap support in FINS analysis while feather ' $\mathrm{A}$ ' and ' $\mathrm{E}$ ' did not amplify for $12 \mathrm{~S} r \mathrm{RNA}$ gene (Figure 1). Feather 'A', 'B', 'C'. 'D' and 'E' were identified using Cytochrome b gene as Lophophorus impejanus, Gallus gallus murghi, Francolinus pondicerianus, Pavo cristatus and Pavo muticus imperator, respectively with strong bootstrap support in FINS analysis while feather ' $F$ ' and 'G' did not amplify for Cytochrome b gene (Figure 2). Overall, FINS analysis of the mitochondrial $12 S$ rRNA and Cytochrome $b$ gene sequences generated from all the analysed species revealed relatively high inter specific variability when compared to intra specific variability.

Sequences of the eight identified species were submitted to the NCBI/GenBank database (Table 2). Interestingly, $12 S r R N A$ partial gene sequence of Ithaginis cruentus and Lophura nycthemera were found to be novel and these sequences were not available on NCBI database prior to our submission. The feathers of these two species were collected from the individuals of known identity.

\begin{tabular}{|c|c|c|c|c|c|}
\hline Feather ID & $12 S$ rRNA & $\begin{array}{c}\text { Reference } \\
\text { Accession no. }\end{array}$ & Cytochrome b & $\begin{array}{c}\text { Reference } \\
\text { Accession no. }\end{array}$ & Species identified using FINS \\
\hline Feather-A & $x$ & --- & $\sqrt{ }$ & gbAF028796.1 & $\begin{array}{c}\text { Himalayan monal } \\
\text { (Lophophorus impejanus) }\end{array}$ \\
\hline Feather-B & $\sqrt{ }$ & gbDQ885561.1 & $\sqrt{ }$ & gbGU261709.1 & $\begin{array}{c}\text { Red Junglefowl } \\
\text { (Gallus gallus murghi) }\end{array}$ \\
\hline Feather-C & $\sqrt{ }$ & gbDQ832103.1 & $\sqrt{ }$ & gbU90648.1 & $\begin{array}{c}\text { Grey francolin } \\
\text { (Francolinus pondicerianus) }\end{array}$ \\
\hline Feather-D & $\sqrt{ }$ & gbAY722396.1 & $\sqrt{ }$ & gbDQ010649.1 & $\begin{array}{l}\text { Indian Peafowl } \\
\text { (Pavo cristatus) }\end{array}$ \\
\hline Feather-E & $x$ & --- & $\sqrt{ }$ & gbDQ010650.1 & $\begin{array}{c}\text { Green Peafowl } \\
\text { (Pavo muticus imperator) }\end{array}$ \\
\hline Feather- $\mathrm{F}^{*}$ & $\sqrt{ }$ & gbEU417810.1 & $x$ & -- & $\begin{array}{c}\text { Silver Pheasant } \\
\text { (Lophura nycthemera) }\end{array}$ \\
\hline Feather-G* & $\sqrt{ }$ & $\mathrm{N} / \mathrm{A}$ & $x$ & --- & $\begin{array}{l}\text { Blood pheasant } \\
\text { (Ithaginis cruentus) }\end{array}$ \\
\hline
\end{tabular}

*feathers collected from the birds of known identity; $\sqrt{ }$ successfully amplified; $X$ not amplified.

Table 1: Identification of forensically informative nucleotide sequencing (FINS) using $12 S$ rRNA and Cytochrome $b$ genes in six galliformes.

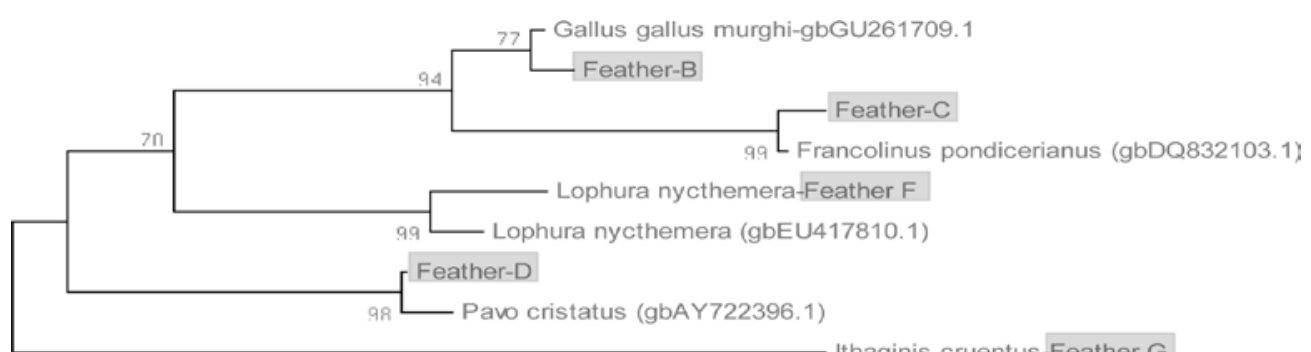

Ithaginis cruentus-Feather $\mathrm{G}$

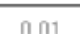

Figure 1: Phylogenetic tree for identification of species from unknown feather samples using 12S rRNA gene by FINS with Kimura-2 parameter distance matrix. 
Citation: Mukesh, Singh SK, Shukla M, Sharma LK, Mohan N, et al. (2013) Identification of Galliformes through Forensically Informative Nucleotide Sequencing (FINS) and its Implication in Wildlife Forensics. J Forensic Res 4: 195 doi:10.4172/2157-7145.1000195

Page 3 of 5

\section{Species specific polymorphic sites in $12 S$ rRNA gene}

In relation to the complete mitochondrial genome of Gallus gallus murghi (GU261709.1), there were 28, 27, 32 and 33 nucleotides substitutions in Ithaginis cruentus, Francolinus pondicerianus, Lophura nycthemera and Pavo cristatus, respectively (Table 3). We found 2 nucleotide additions in Ithaginis cruentus and Francolinus pondicerianus and single nucleotide addition in Lophura nycthemera and Pavo cristatus. Seven deletions were found in Ithaginis cruentus while there was no deletion in Lophura nycthemera.

\section{Species specific polymorphic sites in Cytochrome $b$ gene}

In relation to complete mitochondrial genome of Gallus gallus murghi (GU261709.1), there were 39 and 36 nucleotides substitutions in Francolinus pondicerianus and Pavo muticus imperator, respectively while 37 nucleotides substitutions were found in Lophophorus impejanus and Pavo cristatus (Table 4). Single nucleotide addition was found in Pavo muticus imperator at position 15330 while no addition of nucleotides was found in Francolinus pondicerianus, Lophophorus impejanus and Pavo cristatus. No deletion of nucleotide was observed

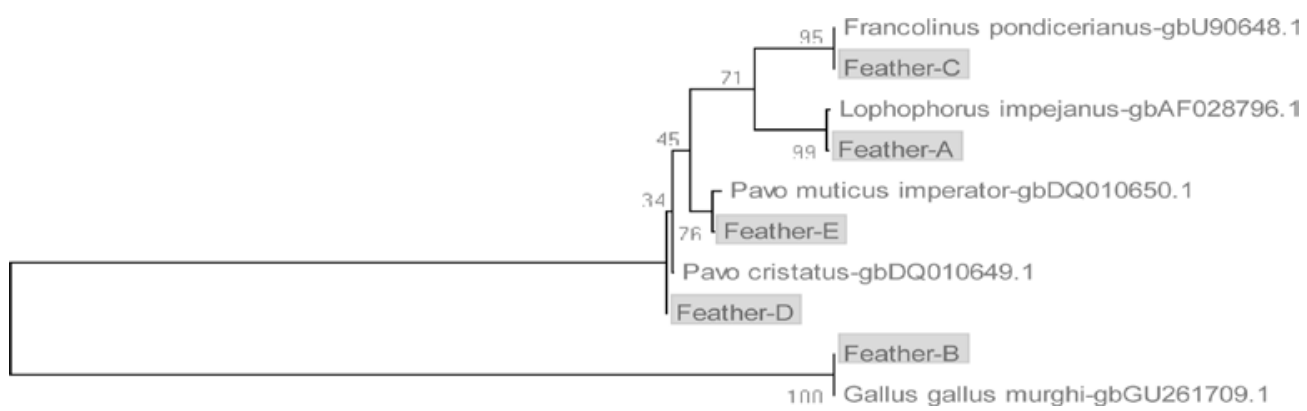

Figure 2: Phylogenetic tree for identification of species from unknown feather samples using Cytochrome $b$ gene by FINS with Kimura-2 parameter distance matrix.

\begin{tabular}{|c|c|}
\hline Species & 12S rRNA (Accession no.) \\
\hline Francolinus pondicerianus & JQ796700 \\
\hline Pavo cristatus & JQ796703 \\
\hline Lophura nycthemera & JQ796702 \\
\hline Ithaginis cruentus & Jytochrome $\boldsymbol{b}$ (Accession no.) \\
\hline Species & JQ796705 \\
\hline Lophophorus impejanus & JQ796704 \\
\hline Francolinus pondicerianus & JQ796706 \\
\hline Pavo cristatus & JQ796707 \\
\hline Pavo muticus imperator & \\
\hline
\end{tabular}

Table 2: Submitted NCBI/GeneBank Accession no for the identified galliformess.

\begin{tabular}{|c|c|c|c|c|c|c|c|c|c|c|c|c|c|c|c|c|c|c|c|c|c|c|}
\hline $\begin{array}{l}\text { Nt Position } \\
\text { Galliformes }\end{array}$ & $\begin{array}{l}1 \\
7 \\
7 \\
8\end{array}$ & $\begin{array}{l}1 \\
7 \\
7 \\
9\end{array}$ & $\begin{array}{l}1 \\
7 \\
8 \\
1\end{array}$ & $\begin{array}{l}1 \\
7 \\
8 \\
3\end{array}$ & $\begin{array}{l}1 \\
7 \\
8 \\
4\end{array}$ & $\begin{array}{l}1 \\
7 \\
8 \\
5\end{array}$ & $\begin{array}{l}1 \\
7 \\
8 \\
6\end{array}$ & $\begin{array}{l}1 \\
7 \\
8 \\
7\end{array}$ & $\begin{array}{l}1 \\
7 \\
9 \\
0\end{array}$ & $\begin{array}{l}1 \\
7 \\
9 \\
1\end{array}$ & $\begin{array}{l}1 \\
7 \\
9 \\
2\end{array}$ & $\begin{array}{l}1 \\
7 \\
9 \\
3\end{array}$ & $\begin{array}{l}1 \\
7 \\
9 \\
4\end{array}$ & $\begin{array}{l}1 \\
7 \\
9 \\
5\end{array}$ & $\begin{array}{l}1 \\
8 \\
0 \\
3\end{array}$ & $\begin{array}{l}1 \\
8 \\
5 \\
2\end{array}$ & $\begin{array}{l}1 \\
8 \\
8 \\
3\end{array}$ & $\begin{array}{l}1 \\
8 \\
9 \\
8\end{array}$ & $\begin{array}{l}1 \\
9 \\
0 \\
3\end{array}$ & $\begin{array}{l}1 \\
9 \\
1 \\
9\end{array}$ & $\begin{array}{l}1 \\
9 \\
2 \\
0\end{array}$ & $\begin{array}{l}1 \\
9 \\
3 \\
9\end{array}$ \\
\hline Gallus gallus murghi & A & $\mathrm{C}$ & $\mathrm{T}$ & C & C & A & $\mathrm{T}$ & $\mathrm{C}$ & A & $\mathrm{C}$ & A & $\mathrm{T}$ & G & $\mathrm{T}$ & $\mathrm{T}$ & C & $\mathrm{T}$ & $\mathrm{T}$ & C & G & C & - \\
\hline Ithaginis cruentus & A & $\mathrm{T}$ & C & A & - & - & A & $\mathrm{T}$ & C & C & A & C & A & $\mathrm{T}$ & $\mathrm{T}$ & $\mathrm{T}$ & C & $\mathrm{T}$ & C & A & C & G \\
\hline Francolinus pondiceranus & A & C & C & $\mathrm{T}$ & C & - & C & C & C & $\mathrm{T}$ & A & $\mathrm{T}$ & C & $\mathrm{T}$ & C & C & $\mathrm{T}$ & C & $\mathrm{C}$ & G & $\mathrm{T}$ & - \\
\hline Lophura nycthemera & - & - & - & - & - & - & - & - & $\mathrm{C}$ & C & A & $\mathrm{T}$ & G & C & C & C & $\mathrm{T}$ & $\mathrm{T}$ & C & A & C & - \\
\hline Pavo cristatus & G & C & C & $\mathrm{T}$ & C & - & A & $\mathrm{T}$ & A & $\mathrm{C}$ & C & $\mathrm{T}$ & A & C & $\mathrm{C}$ & $\mathrm{T}$ & C & C & $\mathrm{T}$ & A & C & - \\
\hline Nt Position & $\begin{array}{l}1 \\
9 \\
4 \\
3\end{array}$ & $\begin{array}{l}1 \\
9 \\
4 \\
8\end{array}$ & $\begin{array}{l}1 \\
9 \\
5 \\
4\end{array}$ & $\begin{array}{l}1 \\
9 \\
5 \\
5\end{array}$ & $\begin{array}{l}1 \\
9 \\
5 \\
7\end{array}$ & $\begin{array}{l}1 \\
9 \\
5 \\
8\end{array}$ & $\begin{array}{l}1 \\
9 \\
5 \\
9\end{array}$ & $\begin{array}{l}1 \\
9 \\
6 \\
1\end{array}$ & $\begin{array}{l}1 \\
9 \\
6 \\
4\end{array}$ & $\begin{array}{l}1 \\
9 \\
6 \\
5\end{array}$ & $\begin{array}{l}1 \\
9 \\
6 \\
9\end{array}$ & $\begin{array}{l}1 \\
9 \\
7 \\
0\end{array}$ & $\begin{array}{l}1 \\
9 \\
7 \\
3\end{array}$ & $\begin{array}{l}1 \\
9 \\
7 \\
5\end{array}$ & $\begin{array}{l}1 \\
9 \\
7 \\
6\end{array}$ & $\begin{array}{l}1 \\
9 \\
7 \\
7\end{array}$ & $\begin{array}{l}1 \\
9 \\
7 \\
8\end{array}$ & $\begin{array}{l}1 \\
9 \\
7 \\
9\end{array}$ & $\begin{array}{l}1 \\
9 \\
8 \\
1\end{array}$ & $\begin{array}{l}1 \\
9 \\
8 \\
2\end{array}$ & $\begin{array}{l}1 \\
9 \\
8 \\
5\end{array}$ & $\begin{array}{l}1 \\
9 \\
8 \\
7\end{array}$ \\
\hline Gallus gallus murghi & C & C & C & $\mathrm{T}$ & A & $\mathrm{T}$ & G & A & A & A & - & C & $\mathrm{T}$ & A & G & C & $\mathrm{T}$ & C & A & $\mathrm{T}$ & C & C \\
\hline Ithaginis cruentus & C & C & $\mathrm{T}$ & A & $\mathrm{T}$ & G & A & A & A & A & A & C & G & A & $\mathrm{T}$ & $\mathrm{T}$ & A & G & $\mathrm{T}$ & C & $\mathrm{T}$ & - \\
\hline Francolinus pondiceranus & $\mathrm{T}$ & C & C & $\mathrm{C}$ & A & $\mathrm{T}$ & G & A & A & A & - & $\mathrm{T}$ & $\mathrm{T}$ & A & G & C & $\mathrm{T}$ & C & A & $\mathrm{T}$ & C & C \\
\hline Lophura nycthemera & C & C & A & A & A & $\mathrm{T}$ & G & G & G & $\mathrm{C}$ & - & C & $\mathrm{T}$ & G & G & C & C & C & A & C & $\mathrm{T}$ & $\mathrm{T}$ \\
\hline Pavo cristatus & C & $\mathrm{T}$ & $\mathrm{C}$ & $\mathrm{T}$ & A & $\mathrm{T}$ & G & A & $A$ & $A$ & - & C & $\mathrm{T}$ & $A$ & $\mathrm{G}$ & C & C & C & $A$ & C & $\mathrm{T}$ & C \\
\hline
\end{tabular}




\begin{tabular}{|c|c|c|c|c|c|c|c|c|c|c|c|c|c|c|c|c|c|c|c|c|c|c|}
\hline Nt Position & $\begin{array}{l}1 \\
9 \\
8 \\
8\end{array}$ & $\begin{array}{l}1 \\
9 \\
8 \\
9\end{array}$ & $\begin{array}{l}1 \\
9 \\
1 \\
0\end{array}$ & $\begin{array}{l}1 \\
9 \\
9 \\
1\end{array}$ & $\begin{array}{l}1 \\
9 \\
9 \\
2 \\
\end{array}$ & $\begin{array}{l}1 \\
9 \\
9 \\
3\end{array}$ & $\begin{array}{l}1 \\
9 \\
9 \\
7\end{array}$ & $\begin{array}{l}1 \\
9 \\
9 \\
8\end{array}$ & $\begin{array}{l}2 \\
0 \\
0 \\
1\end{array}$ & $\begin{array}{l}2 \\
0 \\
0 \\
5\end{array}$ & $\begin{array}{l}2 \\
0 \\
2 \\
0\end{array}$ & $\begin{array}{l}2 \\
0 \\
3 \\
0\end{array}$ & $\begin{array}{l}2 \\
0 \\
5 \\
2\end{array}$ & $\begin{array}{l}2 \\
0 \\
5 \\
4\end{array}$ & $\begin{array}{l}2 \\
0 \\
5 \\
5\end{array}$ & $\begin{array}{l}2 \\
0 \\
6 \\
1\end{array}$ & $\begin{array}{l}2 \\
0 \\
6 \\
3\end{array}$ & $\begin{array}{l}2 \\
0 \\
6 \\
5\end{array}$ & $\begin{array}{l}2 \\
0 \\
6 \\
8\end{array}$ & $\begin{array}{l}2 \\
0 \\
7 \\
4\end{array}$ & $\begin{array}{l}2 \\
0 \\
7 \\
5\end{array}$ & $\begin{array}{l}2 \\
0 \\
7 \\
6\end{array}$ \\
\hline Gallus gallus murghi & C & - & - & $\mathrm{T}$ & C & G & A & $\mathrm{T}$ & G & G & $\mathrm{T}$ & G & A & A & - & C & - & A & A & G & A & C \\
\hline Ithaginis cruentus & - & - & - & - & - & A & G & - & $\mathrm{T}$ & A & - & - & - & - & - & - & - & - & - & - & - & - \\
\hline Francolinus pondiceranus & C & G & C & C & C & G & A & $\mathrm{T}$ & G & G & C & A & A & C & - & $\mathrm{T}$ & - & G & A & G & A & $\mathrm{T}$ \\
\hline Lophura nycthemera & A & - & - & C & C & A & A & C & G & G & C & A & A & A & G & C & - & A & A & G & A & C \\
\hline Pavo cristatus & C & - & - & - & C & A & A & C & G & G & C & A & G & A & - & C & $\mathrm{T}$ & A & G & A & G & C \\
\hline Nt Position & $\begin{array}{l}2 \\
0 \\
8 \\
4\end{array}$ & $\begin{array}{l}2 \\
0 \\
8 \\
5\end{array}$ & $\begin{array}{l}2 \\
0 \\
8 \\
6\end{array}$ & $\begin{array}{l}2 \\
0 \\
8 \\
7\end{array}$ & $\begin{array}{l}2 \\
0 \\
9 \\
2\end{array}$ & $\begin{array}{l}2 \\
0 \\
9 \\
7\end{array}$ & $\begin{array}{l}2 \\
1 \\
0 \\
8\end{array}$ & $\begin{array}{l}2 \\
1 \\
1 \\
7\end{array}$ & $\begin{array}{l}2 \\
1 \\
1 \\
8\end{array}$ & $\begin{array}{l}2 \\
1 \\
2 \\
1\end{array}$ & $\begin{array}{l}2 \\
1 \\
2 \\
4\end{array}$ & $\begin{array}{l}2 \\
1 \\
2 \\
5\end{array}$ & $\begin{array}{l}2 \\
1 \\
2 \\
7\end{array}$ & $\begin{array}{l}2 \\
8 \\
2 \\
9\end{array}$ & $\begin{array}{l}2 \\
1 \\
3 \\
0\end{array}$ & $\begin{array}{l}2 \\
1 \\
3 \\
1\end{array}$ & $\begin{array}{l}2 \\
1 \\
3 \\
3\end{array}$ & $\begin{array}{l}2 \\
1 \\
3 \\
7\end{array}$ & $\begin{array}{l}2 \\
1 \\
3 \\
8\end{array}$ & & & \\
\hline Gallus gallus murghi & C & G & C & A & G & C & G & A & $\mathrm{T}$ & $\mathrm{T}$ & A & C & C & C & C & $\mathrm{T}$ & A & $\mathrm{T}$ & C & & & \\
\hline Ithaginis cruentus & - & - & - & - & - & - & - & - & - & - & - & - & - & - & - & - & - & - & - & & & \\
\hline Francolinus pondiceranus & C & C & $\mathrm{T}$ & G & A & $\mathrm{T}$ & A & A & C & $\mathrm{T}$ & G & C & C & C & - & - & - & - & - & & & \\
\hline Lophura nycthemera & C & G & $\mathrm{T}$ & G & G & C & G & G & C & $\mathrm{T}$ & A & $\mathrm{T}$ & $\mathrm{T}$ & $\mathrm{T}$ & $\mathrm{T}$ & C & $\mathrm{T}$ & C & $\mathrm{T}$ & & & \\
\hline Pavo cristatus & $\mathrm{T}$ & G & C & A & G & C & A & G & C & C & A & $T$ & C & C & C & C & - & - & - & & & \\
\hline
\end{tabular}

Table 3: Haplotypes and polymorphic sites in galliformes using combined analysis of 12S rRNA gene with complete mitochondrial genome of G.g.murghi (GU261709.1).

\begin{tabular}{|c|c|c|c|c|c|c|c|c|c|c|c|c|c|c|c|c|c|c|c|c|c|c|}
\hline Nt Position & $\begin{array}{l}1 \\
5 \\
0 \\
1 \\
1\end{array}$ & $\begin{array}{l}1 \\
5 \\
0 \\
1 \\
2\end{array}$ & $\begin{array}{l}1 \\
5 \\
0 \\
1 \\
8\end{array}$ & $\begin{array}{l}1 \\
5 \\
0 \\
2 \\
0\end{array}$ & $\begin{array}{l}1 \\
5 \\
0 \\
2 \\
6\end{array}$ & $\begin{array}{l}1 \\
5 \\
0 \\
2 \\
7\end{array}$ & $\begin{array}{l}1 \\
5 \\
0 \\
2 \\
8\end{array}$ & $\begin{array}{l}1 \\
5 \\
0 \\
2 \\
9\end{array}$ & $\begin{array}{l}1 \\
5 \\
0 \\
3 \\
2\end{array}$ & $\begin{array}{l}1 \\
5 \\
0 \\
3 \\
9\end{array}$ & $\begin{array}{l}1 \\
5 \\
0 \\
4 \\
4\end{array}$ & $\begin{array}{l}1 \\
5 \\
0 \\
5 \\
2\end{array}$ & $\begin{array}{l}1 \\
5 \\
0 \\
6 \\
1\end{array}$ & $\begin{array}{l}1 \\
5 \\
0 \\
6 \\
3\end{array}$ & $\begin{array}{l}1 \\
5 \\
0 \\
6 \\
4\end{array}$ & $\begin{array}{l}1 \\
5 \\
0 \\
7 \\
0\end{array}$ & $\begin{array}{l}1 \\
5 \\
0 \\
7 \\
3\end{array}$ & $\begin{array}{l}1 \\
5 \\
0 \\
8 \\
2\end{array}$ & $\begin{array}{l}1 \\
5 \\
0 \\
8 \\
5\end{array}$ & $\begin{array}{l}1 \\
5 \\
0 \\
8 \\
8\end{array}$ & $\begin{array}{l}1 \\
5 \\
1 \\
0 \\
0\end{array}$ & $\begin{array}{l}1 \\
5 \\
1 \\
0 \\
7\end{array}$ \\
\hline Gallus gallus murghi & A & $\mathrm{T}$ & G & C & C & A & $\mathrm{T}$ & G & C & C & C & A & C & $\mathrm{T}$ & G & C & A & A & C & A & C & C \\
\hline Francolinus pondicerianus & - & - & - & - & - & - & - & - & C & $\mathrm{C}$ & C & $\mathrm{C}$ & A & C & $A$ & $\mathrm{~T}$ & A & $\mathrm{T}$ & $\mathrm{T}$ & C & C & $\mathrm{T}$ \\
\hline Lophophorus impejanus & C & C & A & A & $\mathrm{C}$ & A & $\mathrm{T}$ & $\mathrm{C}$ & C & $\mathrm{C}$ & $\mathrm{T}$ & $\mathrm{C}$ & $\mathrm{T}$ & $\mathrm{T}$ & A & C & C & $\mathrm{C}$ & C & C & $\mathrm{T}$ & C \\
\hline Pavo cristatus & $A$ & C & G & A & $\mathrm{T}$ & G & $\mathrm{C}$ & $\mathrm{C}$ & $\mathrm{T}$ & $A$ & $\mathrm{~T}$ & $A$ & A & $\mathrm{T}$ & A & C & $\mathrm{C}$ & $A$ & C & A & A & C \\
\hline Pavo muticus imperator & G & C & G & A & $\mathrm{T}$ & G & $\mathrm{C}$ & C & $\mathrm{T}$ & A & $\mathrm{T}$ & A & A & $\mathrm{T}$ & A & C & C & A & C & A & A & C \\
\hline Galliformes & $\begin{array}{l}1 \\
5 \\
1 \\
0 \\
9\end{array}$ & $\begin{array}{l}1 \\
5 \\
1 \\
1 \\
2\end{array}$ & $\begin{array}{l}1 \\
5 \\
1 \\
1 \\
5\end{array}$ & $\begin{array}{l}1 \\
5 \\
1 \\
1 \\
8\end{array}$ & $\begin{array}{l}1 \\
5 \\
1 \\
2 \\
1\end{array}$ & $\begin{array}{l}1 \\
5 \\
1 \\
2 \\
4\end{array}$ & $\begin{array}{l}1 \\
5 \\
1 \\
2 \\
7\end{array}$ & $\begin{array}{l}1 \\
5 \\
1 \\
3 \\
9\end{array}$ & $\begin{array}{l}1 \\
5 \\
1 \\
4 \\
5\end{array}$ & $\begin{array}{l}1 \\
5 \\
1 \\
4 \\
8\end{array}$ & $\begin{array}{l}1 \\
5 \\
1 \\
5 \\
1\end{array}$ & $\begin{array}{l}1 \\
5 \\
1 \\
5 \\
4\end{array}$ & $\begin{array}{l}1 \\
5 \\
1 \\
6 \\
0\end{array}$ & $\begin{array}{l}1 \\
5 \\
1 \\
6 \\
3\end{array}$ & $\begin{array}{l}1 \\
5 \\
1 \\
6 \\
6\end{array}$ & $\begin{array}{l}1 \\
5 \\
1 \\
8 \\
1\end{array}$ & $\begin{array}{l}1 \\
5 \\
1 \\
8 \\
4\end{array}$ & $\begin{array}{l}1 \\
5 \\
1 \\
9 \\
3\end{array}$ & $\begin{array}{l}1 \\
5 \\
1 \\
9 \\
9\end{array}$ & $\begin{array}{l}1 \\
5 \\
2 \\
0 \\
5\end{array}$ & $\begin{array}{l}1 \\
5 \\
2 \\
0 \\
6\end{array}$ & $\begin{array}{l}1 \\
5 \\
2 \\
1 \\
1\end{array}$ \\
\hline Gallus gallus murghi & C & $\mathrm{T}$ & $\mathrm{C}$ & G & $\mathrm{C}$ & A & A & $\mathrm{C}$ & G & $\mathrm{T}$ & C & $\mathrm{C}$ & C & C & $\mathrm{C}$ & C & $\mathrm{T}$ & $\mathrm{T}$ & $\mathrm{C}$ & A & G & A \\
\hline Francolinus pondicerianus & $\mathrm{T}$ & A & $\mathrm{C}$ & G & C & A & G & A & A & $\mathrm{C}$ & C & $\mathrm{T}$ & C & C & C & C & C & $\mathrm{T}$ & $\mathrm{T}$ & A & G & A \\
\hline Lophophorus impejanus & C & A & $\mathrm{C}$ & A & $\mathrm{T}$ & $\mathrm{T}$ & A & $\mathrm{C}$ & A & $\mathrm{T}$ & $\mathrm{C}$ & $\mathrm{T}$ & $\mathrm{C}$ & C & $\mathrm{C}$ & C & $\mathrm{C}$ & $\mathrm{T}$ & $\mathrm{C}$ & C & A & C \\
\hline Pavo cristatus & C & A & $\mathrm{T}$ & A & $\mathrm{C}$ & A & A & C & A & $\mathrm{T}$ & $\mathrm{T}$ & $\mathrm{T}$ & C & A & $\mathrm{T}$ & $\mathrm{T}$ & C & C & $\mathrm{T}$ & C & G & A \\
\hline Pavo muticus imperator & C & A & $\mathrm{T}$ & A & $\mathrm{C}$ & $A$ & A & $\mathrm{C}$ & G & $\mathrm{T}$ & $\mathrm{T}$ & $\mathrm{T}$ & $\mathrm{T}$ & A & $\mathrm{T}$ & C & $\mathrm{C}$ & C & $\mathrm{C}$ & C & G & $A$ \\
\hline Galliformes & $\begin{array}{l}1 \\
5 \\
2 \\
1 \\
7\end{array}$ & $\begin{array}{l}1 \\
5 \\
2 \\
2 \\
7\end{array}$ & $\begin{array}{l}1 \\
5 \\
2 \\
2 \\
9\end{array}$ & $\begin{array}{l}1 \\
5 \\
2 \\
3 \\
0\end{array}$ & $\begin{array}{l}1 \\
5 \\
2 \\
3 \\
2\end{array}$ & $\begin{array}{l}1 \\
5 \\
2 \\
3 \\
5\end{array}$ & $\begin{array}{l}1 \\
5 \\
2 \\
3 \\
8\end{array}$ & $\begin{array}{l}1 \\
5 \\
2 \\
4 \\
7\end{array}$ & $\begin{array}{l}1 \\
5 \\
2 \\
5 \\
7\end{array}$ & $\begin{array}{l}1 \\
5 \\
2 \\
5 \\
9\end{array}$ & $\begin{array}{l}1 \\
5 \\
2 \\
7 \\
1\end{array}$ & $\begin{array}{l}1 \\
5 \\
2 \\
7 \\
4\end{array}$ & $\begin{array}{l}1 \\
5 \\
2 \\
8 \\
0\end{array}$ & $\begin{array}{l}1 \\
5 \\
2 \\
8 \\
3\end{array}$ & $\begin{array}{l}1 \\
5 \\
2 \\
8 \\
6\end{array}$ & $\begin{array}{l}1 \\
5 \\
2 \\
8 \\
9\end{array}$ & $\begin{array}{l}1 \\
5 \\
2 \\
9 \\
2\end{array}$ & $\begin{array}{l}1 \\
5 \\
2 \\
9 \\
5\end{array}$ & $\begin{array}{l}1 \\
5 \\
2 \\
9 \\
8\end{array}$ & $\begin{array}{l}1 \\
5 \\
3 \\
0 \\
1\end{array}$ & $\begin{array}{l}1 \\
5 \\
3 \\
0 \\
4\end{array}$ & $\begin{array}{l}1 \\
5 \\
3 \\
1 \\
0\end{array}$ \\
\hline Gallus gallus murghi & C & C & $\mathrm{C}$ & $\mathrm{T}$ & $\mathrm{C}$ & G & A & $\mathrm{C}$ & A & $\mathrm{C}$ & A & $\mathrm{C}$ & $\mathrm{C}$ & C & $\mathrm{C}$ & $\mathrm{T}$ & G & C & $\mathrm{T}$ & $\mathrm{T}$ & C & G \\
\hline Francolinus pondicerianus & $\mathrm{T}$ & $\mathrm{T}$ & G & C & $\mathrm{C}$ & A & A & $\mathrm{T}$ & A & $\mathrm{C}$ & A & $\mathrm{C}$ & $A$ & $\mathrm{~T}$ & $\mathrm{~T}$ & C & A & C & $\mathrm{T}$ & C & $\mathrm{T}$ & $A$ \\
\hline Lophophorus impejanus & $\mathrm{T}$ & C & A & $\mathrm{T}$ & $\mathrm{T}$ & G & G & $\mathrm{C}$ & A & $\mathrm{C}$ & A & $\mathrm{T}$ & A & $\mathrm{T}$ & $\mathrm{T}$ & C & A & A & $\mathrm{T}$ & C & C & A \\
\hline Pavo cristatus & C & $\mathrm{T}$ & A & $\mathrm{T}$ & $\mathrm{C}$ & A & A & $\mathrm{C}$ & A & C & G & C & A & C & $\mathrm{C}$ & C & A & C & $\mathrm{C}$ & A & C & A \\
\hline Pavo muticus imperator & $\mathrm{C}$ & $\mathrm{T}$ & A & $\mathrm{T}$ & $\mathrm{C}$ & A & A & $\mathrm{C}$ & G & $\mathrm{T}$ & $A$ & $\mathrm{C}$ & A & C & $\mathrm{C}$ & C & A & C & $T$ & A & C & A \\
\hline Nt Position & $\begin{array}{l}1 \\
5 \\
3 \\
1 \\
3\end{array}$ & $\begin{array}{l}1 \\
5 \\
3 \\
3 \\
0\end{array}$ & $\begin{array}{l}1 \\
5 \\
3 \\
3 \\
9\end{array}$ & $\begin{array}{l}1 \\
5 \\
3 \\
4 \\
5\end{array}$ & $\begin{array}{l}1 \\
5 \\
3 \\
6 \\
9\end{array}$ & $\begin{array}{l}1 \\
5 \\
3 \\
7 \\
8\end{array}$ & $\begin{array}{l}1 \\
5 \\
3 \\
8 \\
4\end{array}$ & $\begin{array}{l}1 \\
5 \\
3 \\
8 \\
8\end{array}$ & $\begin{array}{l}1 \\
5 \\
3 \\
9 \\
6\end{array}$ & & & & & & & & & & & & & \\
\hline Gallus gallus murghi & $\mathrm{C}$ & - & $\mathrm{C}$ & $\mathrm{T}$ & $\mathrm{T}$ & $\mathrm{T}$ & $\mathrm{C}$ & $\mathrm{C}$ & G & & & & & & & & & & & & & \\
\hline Francolinus pondicerianus & $\mathrm{C}$ & - & $\mathrm{T}$ & C & $\mathrm{C}$ & $\mathrm{C}$ & $A$ & $\mathrm{~T}$ & A & & & & & & & & & & & & & \\
\hline Lophophorus impejanus & A & - & - & - & - & - & - & - & - & & & & & & & & & & & & & \\
\hline Pavo cristatus & A & - & - & - & - & - & - & - & - & & & & & & & & & & & & & \\
\hline Pavo muticus imperator & $A$ & C & - & - & - & - & - & - & - & & & & & & & & & & & & & \\
\hline
\end{tabular}

(Polymorphic sites between Indian peafowl and green peafowl are highlighted in check box)

Table 4: Haplotypes and polymorphic sites in galliformes using combined analysis of Cytochrome $b$ gene with complete mitochondrial genome of G.g.murghi (GU261709.1). 
in any of the studied galliform species. We found nine polymorphic sites between Pavo cristatus and Pavo muticus imperator on positions $15011,15145,15160,15181,15199,15257,15259,15271$ and 15330 in Cytochrome $b$ gene and these sites can be used to differentiate Pavo cristatus to Pavo muticus imperator.

Our results showed the applicability of shed feathers to amplify the mitochondrial gene and the potential of FINS technology in identifying the species. However, the homologous sequences from NCBI/GenBank should be retrieved with caution as this may often give erroneous results when the sequence of the species in question is not available in the NCBI database and subsequently there is a high possibility that the sequence of the closely related species may be retrieved. We recommend generating sequence for more than one gene for the sample in question and then finding homologous sequences for each gene on NCBI/GenBank database. This way, one can minimize the possibility of retrieving wrong sequences and subsequently decrease the chances of misidentification the species in question. In FINS analysis, identification of unknown sample can be performed when the sequence of a gene from unknown sample is introduced in the estimation of genetic distance among a set of reference sequence and draw a dendogram based on the distance matrix. In this parsimony (FINS) method the unknown sample will cluster more closely with the same species [11]. This approach will be particularly useful for wildlife forensics to identify the species from feathers otherwise morphometric identification of the species using feathers or meat is confusing, inaccurate and needs expertise.

\section{Acknowledgements}

We thank Director, Dean and Research Coordinator, Wildlife Institute of India, Dehradun, Uttarakhand. We acknowledge the support received from lab members at Wildlife Forensic Cell, WII, Dehradun. Special thanks are due to Shri Sandeep Kumar Gupta (WII) and Dr. Vipin Sharma (LaCONES, CCMB) to review the previous drafts of the present manuscript. Authors are thanking to Dr. Amit Kotia for providing feathers of blood pheasant. The research was the supplementary outcome of a national project on 'Conservation of Red Junglefowl in India' and funded by Wildlife Institute of India, Dehradun.

\section{References}

1. Keane A, Brooke MdeL, McGowan PJK (2005) Correlates of extinction risk and hunting pressure in game birds (Galliformes). Biol Conserv126: 216-223.

2. Sathyakumar S, Sivakumar K (2007) The Galliformes of India: An Introduction. Wildlife Institute of India, Dehradun, India.
3. Saferstain R (1982) Forensic sciences handbook. Englewood Cliffs, $\mathrm{NJ}$ : Prentice Hall.

4. Forrest AR, Carnegie PR (1994) Identification of gourmet meat using FINS (forensically informative nucleotide sequencing). Biotechniques 17: 24-26.

5. Soteriou B, Fisher RA, Khan IM, Kessling AM, Archard LC, et al. (1995) Conserved gene sequences for species identification: PCR analysis of the 3' UTR of the SON gene distinguishes human and other mammalian DNAs. Forensic Sci Int 73: 171-181.

6. Carrera E, Garcia T, Cespedes A, Gonzalez I, Fernandez A, et al. (1999) Salmon and trout analysis by PCR-RFLP for identity authentication. J Food Sci 64: 410-413.

7. Chapela MJ, Sotelo CG, Mata PC, Perez-martin RI, Rehbein H, et al. (2002) Identification of cephalopod species (ommastrephidae and loliginidae) in seafood products by forensically informative nucleotide sequencing (FINS). J Food Sci 67: 1672-1676

8. Gupta SK, Verma SK, Singh L (2005) Molecular insight into a wildlife crime: the case of a peafowl slaughter. Forensic Sci Int 154: 214-217.

9. Vences M, Thomas M, Meijden AVD, Chiari Y, Vieites DR (2005) Comparative performance of the $16 \mathrm{~S}$ rRNA gene in DNA barcoding of amphibians. Front Zool 2: 5.

10. Sahajpal V, Goyal SP (2010) Identification of a forensic case using microscopy and forensically informative nucleotide sequencing (FINS): A case study of small Indian civet (Viverricula indica). Sci Justice 50: 94-97.

11. Bartlett SE, Davidson WS (1992) FINS (forensically informative nucleotide sequencing): a procedure for identifying the animal origin of biological specimens. Biotechniques 12: 408-411.

12. Guha S, Kashyap VK (2006) Molecular identification of lizard by RAPD and FINS of mitochondrial 16s rRNA gene. Leg Med (Tokyo) 8: 5-10.

13. Thakur M, Rai ID, Mandhan RP, Sathyakumar S (2011) A panel of polymorphic microsatellite markers in Himalayan monal Lophophorus impejanus developed by cross-species amplification and their applicability in other Galliformes. Eur J Wild Res 57: 983-989.

14. Kocher TD, Thomas WK, Meyer A, Edwards SV, Pääbo S, et al. (1989) Dynamics of mitochondrial DNA evolution in animals: amplification and sequencing with conserved primers. Proc Natl Acad Sci U S A 86: 6196-6200.

15. Hall TA (1999) BioEdit: a user-friendly biological sequence alignment editor and analysis program for windows 95/98/NT. Nucleic Acids Symposium Series 41: $95-98$.

16. Tamura K, Peterson D, Peterson N, Stecher G, Nei M, et al. (2011) MEGA5 molecular evolutionary genetics analysis using maximum likelihood evolutionary distance, and maximum parsimony methods. Mol Biol Evol 28 2731-2739. 